\title{
PERFORMANCE PREDICTION OF GSM DIGITAL CELLULAR SPEECH AND IMAGE TRANSMISSION
}

\author{
E.A. Quincy and R.J. Achatz \\ Institute for Telecommunication Sciences \\ National Telecommunication and Information Administration \\ U.S. Department of Commerce \\ Boulder, CO 80303 U.S.A \\ equincy@ntia.its.bldrdoc.gov
}

\begin{abstract}
The Group Special Mobile (GSM) digital cellular system provides the European countries with a standard land mobile radio (LMR) architecture. This paper illustrates how voice coded (vocoded) speech and uncompressed image transmission can be expected to degrade as a function of signal to noise ratio (SNR) and carrier to interference ratio (CIR) for a cochannel GSM interferer. Performance is predicted by Monte Carlo simulation of the system. This simulated GSM system employed (1) a Gaussian minimum shift keying (GMSK) modem with convolutional coding and Viterbi decoding, (2) a fivepath urban channel impulse response with additive white Gaussian noise (AWGN), (3) a minimum mean square error (MMSE) equalizer, and (4) limiterdiscriminator detection. These results demonstrate that GSM systems in an urban environment can transport useable speech and images without packet retransmission provided the $\mathrm{SNR} \geq 8 \mathrm{~dB}$ and $\mathrm{CIR} \geq 8$ dB.
\end{abstract}

\section{INTRODUCTION}

The Group Special Mobile (GSM) digital cellular system provides European countries with a standard land mobile radio (LMR) architecture for communications [1]. Benelli, et al [2] estimated the bit error ratio (BER) as a function of signal to noise ratio (SNR) and parametrically as a function of three typical channels, including an urban channel, for a GSM system employing a Viterbi demodulator and decoder. Varashney, et al [3] computed BER for a LMR system employing Gaussian minimum shift keying (GMSK) as a function of the IF filter bandwidth and parametrically as a function of SNR, carrier to interference ratio (CIR), and system environment.

Our objective was to determine the effects of multipath, noise, and cochannel interference on the performance of GSM digital cellular systems using convolutional coding and Viterbi decoding. Performance was expressed in terms of BER, voice coded (vocoded) speech quality, and uncompressed image quality. A typical five-path impulse response, derived from measurements made by the Institute for Telecommunication Sciences (ITS) in an urban environment, provided the multipath distortion. Cochannel interference was modelled as other fringe GSM systems reusing the same frequency. The BER, speech quality, and image quality were predicted as a function of SNR and CIR for received signals operating in this environment.

ITS has previously investigated [4] similar effects of channel and system parameters on performance of LMR, wireless private branch exchange, wireless local area networks, and ionospheric high frequency systems.

In this paper we demonstrate how the performance of the GSM system described in Fig. 1 is expected to degrade as a function of SNR and CIR for a cochannel GSM interferer. This simulated GSM system employs (1) a GMSK modem with convolutional coding and Viterbi decoding, (2) a typical measured urban impulse response channel with additive white Gaussian noise (AWGN), (3) a minimum mean square error (MMSE) equalizer, and (4) limiter-discriminator detection.

\section{DESCRIPTION OF METHOD}

\section{A. Simulation}

The results in this paper were obtained with Monte-Carlo techniques using a commercial software communications system simulation tool enhanced with modules developed by ITS [4]. This tool allows configuration and testing of a communication system with modular transmitter, channel, and receiver models in a graphical user interface (GUI) environment. The system configuration was then saved in a text file for execution as a batch job.

Our main objective was to relate the degradation of digital sources transmitted by a GSM modem to the radio system and channel parameters (BER, SNR, and CIR). We performed a Monte Carlo simulation of the entire system and channel for 79 pairs of SNR and CIR. Then we plotted the estimated BER as a function of SNR and CIR. Bit 
error streams for the radio link were collected for each combination of SNR and CIR. The error streams are the result of synchronously combining the transmitted bit streams with the detected bit streams using the exclusive OR operation. These error streams contain all the error bursts and error gaps generated by the link. The error streams were then used to distort digital speech and image sources in "near real-time," again using the exclusive OR operation.

\section{B. Channel Distortion}

Transmitted symbols were distorted by multipath and cochannel interference. Multipath distortion was derived from impulse response measurements performed by ITS. The measurements were taken with the patented ITS digital sampling channel probe [5]. The channel probe's measurement time resolution is $40 \mathrm{~ns}$ and maximum measurable delay spread is $20.44 \mu \mathrm{s}$. The representative 5-path impulse response measurement was taken from a semiresidential street (near downtown Boulder, $\mathrm{CO}$ ) with a mixture of one- and two-story buildings. The maximum delay spread (time difference of last and first path) for the 5 path channel was approximately one-half of a symbol period $(1.85 \mu \mathrm{s})$. The transfer function magnitude was normalized to a maximum of unity.

The impulse response was measured over a much wider bandwidth than that occupied by the transmitter. Therefore, it could be decimated without significant degradation. It was decimated from 370 samples/symbol to 32 samples/symbol. A finite impulse response (FIR) filter module was customized by ITS to model the decimated impulse response for convolution with the transmitted signal. In order to efficiently implement the channel effect using the FFT, the long transmitted signal was segmented, and the overlap and add method [6] was employed to perform the convolution. Cochannel interference was introduced by an independent GSM modem. This interfering modem transmitted a random data stream (uncorrelated with the desired transmitter source) through a channel without multipath or other degradation.

\section{Sources}

A random data stream of up to 500,000 symbols (bits) was employed as the source in the system simulations to obtain estimates of the BER and collect bit error streams. The bit error streams were then used to degrade speech and images off-line. Speech files from the TIMIT standard source (distributed by the National Institute for Standards and Technology) were used to evaluate speech quality. The sentence of speech used ("Rob sat by the pond and sketched stray geese") is about 3.4 seconds long and was recorded at 16 kilosamples per second (ksps) with 16 bit resolution at $256 \mathrm{kbps}$. This $256 \mathrm{kbps}$ speech file was decimated to $8 \mathrm{ksps}$ and 8 bit resolution to produce "toll quality" $64 \mathrm{kbps}$ PCM speech and was processed by the U.S. Federal Standard 1016, 4.8 kbps Code Excited Linear Prediction (CELP) Vocoding Algorithm [7]. The vocoded data stream was combined with the error sequence derived from the simulation using the exclusive $\mathrm{OR}$ operation and decoded back into $64 \mathrm{kbps}$ PCM speech. The quality of the speech was then evaluated using an $8 \mathrm{ksps}, 8$ bit personal computer soundboard and speakers.

A U.S. Geological Survey topographic map was scanned and used as the image source. This image was chosen because it contained easily identifiable features such as shading, sharp lines, symbols, and text. The image was derived from a standard bit map file. The picture size was limited to 320 pixels by 200 lines in order to limit execution time. Pixel resolution was limited to 16 gray shades represented by 4 bits.

\section{Performance Prediction}

The Institute has been developing objective speech $[8,9]$ and video $[10,11]$ quality measurement software in support of various standards groups for a number of years. Until recently, this software was designed to primarily measure speech degradations from telephony channels. Current efforts are directed towards development of objective measurements that are sensitive to radio channel degradations on speech quality. Development of objective measurements robust to a wide variety of system and channel degradations is extremely difficult and timeconsuming. For expediency, preliminary speech and image quality ratings presented here were based on consensus scores from a two person subjective panel. These subjective ratings represent an opinion class scale from one to five with five being the best. With large rating panels, the ratings would usually be quoted in terms of the mean opinion score or MOS.

\section{EXPERIMENTAL DESCRIPTION}

A block diagram of the simulated GSM system is shown in Fig. 1. The modem uses GMSK with a data rate of $270.833 \mathrm{kbps}$ ( $3.692 \mu$ s symbol period). The Gaussian pulse extended over 3 symbol periods and had a timebandwidth product of 0.3 . Limiter-discriminator detection was used. A 6-pole Butterworth filter with a $3 \mathrm{~dB}$ bandwidth of $270 \mathrm{kHz}$ was used to reduce noise at the discriminator input. Although GSM operates in the 900 $\mathrm{MHz}$ band, simulation was conducted at baseband as is commonly done. 
A half-rate convolutional code complemented with a Viterbi decoder was used to compensate for intersymbol interference introduced by the extended pulse shape and residual multipath effects after equalization. The decoder had a length of 5-symbol periods. A MMSE equalizer was used to minimize channel multipath distortion. The channel impulse response was used in the equalizer design but it had to equalize in the presence of noise and interference. The equalizer was intended to provide an upper bound on performance of other equalizer implementations that require sounding of the channel.

\section{EXPERIMENTAL PERFORMANCE PREDICTIONS}

Results of the performance prediction are provided for random data streams, vocoded speech, and uncompressed image transmission. Fig. 2 and 3 show the estimated BER as a function of SNR and CIR. An AWGN curve was included for reference in both figures representing GSM performance without channel distortion or cochannel interference. In addition, these figures show the regions of speech and image quality, respectively. These regions are partitioned with dashed lines and labeled with the quality class (boxed numbers ranging from 1-5) from the opinion scores. The BER curves for this measured 5-path urban channel did not exhibit channel limiting as SNR increased. The curves are similar in shape to that shown by Benelli, et al [2] for a GSM system operating in an urban channel. In Fig. 2 the center of Class 5 operation for vocoded speech transmission is at a SNR of $14 \mathrm{~dB}$ and a CIR of 10 dB. Similarly, the center of Class 2 speech transmission is at a SNR and CIR of $8 \mathrm{~dB}$. Class 2 is the lowest useful class that contains some intelligible speech but the speaker is not recognizable. Fig. 4 illustrates Classes 2 and 5 received speech waveforms. Class 2 exhibits what corresponds to audible burps when error bursts are longer than can be corrected by vocoding.

Fig. 3 shows image quality classes overlaid on the BER curves. The center of Class 5 image transmission is at a SNR of $13 \mathrm{~dB}$ and a CIR of $10 \mathrm{~dB}$. The center of Class 2 image transmission is at a SNR of $5 \mathrm{~dB}$ and a CIR of 8 $\mathrm{dB}$. This class contains significantly distorted images that still have useful detail. Similar to Fig. 4, Fig. 5 illustrates representative image quality transmission classes. Generally images are more tolerable to bit errors than speech since they have built in two-dimensional redundancy compared to one-dimensional redundancy in speech. This is evidenced comparing Fig. 3 to Fig. 2; image class boundaries shifted to higher BER values than exhibited by speech.

\section{CONCLUSION}

Data, vocoded speech, and uncompressed image transmission degradation was explored for a GSM system operating in a 5-path urban channel with GSM cochannel interference and AWGN using Monte Carlo simulation. The simulated GSM system employed a GMSK modem with convolutional coding and Viterbi decoding, a channel representing typical measured impulse responses, a MMSE equalizer, and limiter-discriminator detection. Predicted BER, speech quality class, and image quality class were all shown as a function of SNR and CIR. Equalization and coding mitigated channel distortion as evidenced by BER curves not being channel limited at high SNR. Minimum useable speech transmission occurs when system and channel parameters are centered around a SNR and CIR of $8 \mathrm{~dB}$. Similarly, minimum useable image transmission occurs when these parameters are centered around a SNR of $6 \mathrm{~dB}$ and a CIR of $8 \mathrm{~dB}$. Example speech waveforms and images were provided for Classes 2 and 5.

\section{ACKNOWLEDGMENT}

The authors express their gratitude to J. M. Ratzlof for her diligent efforts in constructing figures for this paper. Technical and editorial reviews by J. F. Mastrangelo, J. A. Wepman, and N. Sexton were especially useful.

\section{REFERENCES}

[1] D.J. Goodman, "Trends in cellular and cordless communications," IEEE Communication Magazine, pp. 3140, June 1991

[2] G. Benelli, A. Garzelli, and F. Salvi, "Simplified Viterbi processors for the GSM Pan-European cellular communication system," IEEE Trans. on Vehicular Technology, vol. 43, pp. 870-878, Nov. 1994.

[3] J. Varashney, and J.E. Salt, "BER analysis of GMSK with differential detection in a land mobile channel," IEEE Trans. on Vehicular Technology, vol. 42, pp. 683-689, Nov. 1993.

[4] E.A. Quincy, and R.J. Achatz, "Wireless performance prediction via software simulation," Proc. of Wireless $94,6 \mathrm{th}$ Internat. Conf. on Wireless Commun., pp.324-343, July 1994

[5] J.A. Wepman, J.R. Hoffman, and L.H. Loew, "Impulse Response Measurements in the $1850-1990 \mathrm{MHz}$ Band in Large Cells," NTIA Report 94-309, June 1994. (NTIS Order No. PB 94204906)

[6] M.C. Jeruchim, P. Balaban, and K.S. Shanmugan, Simulation of Communication Systems. New York, NY: Plenum Press, pp. 102-105, 1992.

[7] J.P. Campbell, "The DOD 4.8 KBPS Standard," Advances in Speech Coding, Klower Academic Publishers, 1990.

[8] E. A. Quincy, "Prolog-based expert pattern recognition system shell for technology independent, user-oriented classification of voice transmission quality", Proc. IEEE International Conference on Communications, pp. 1164-1171, June 1987.

[9] R. Kubichek, D.Atkinson, and A.Webster, "Advances in objective voice quality assessment", in Proc. IEEE Global 
Telecommunications Conference, vol. 3, pp. 1765-1770, Dec. 1991.

[10] E.A. Quincy, "Gradient measures of compressed video teleconferencing quality," Proc. IEEE Pacific Rim Conference Communications, Computers and Signal Processing, pp. 356354, May 1991.
[11] S. Wolf, M.H. Pinson, S.D. Voran, and A.A. Webster, "Objective quality assessment of digitally transmitted video," Proc. IEEE Pacific Rim Conference Communications, Computers and Signal Processing, pp. 478-489, May 1991.

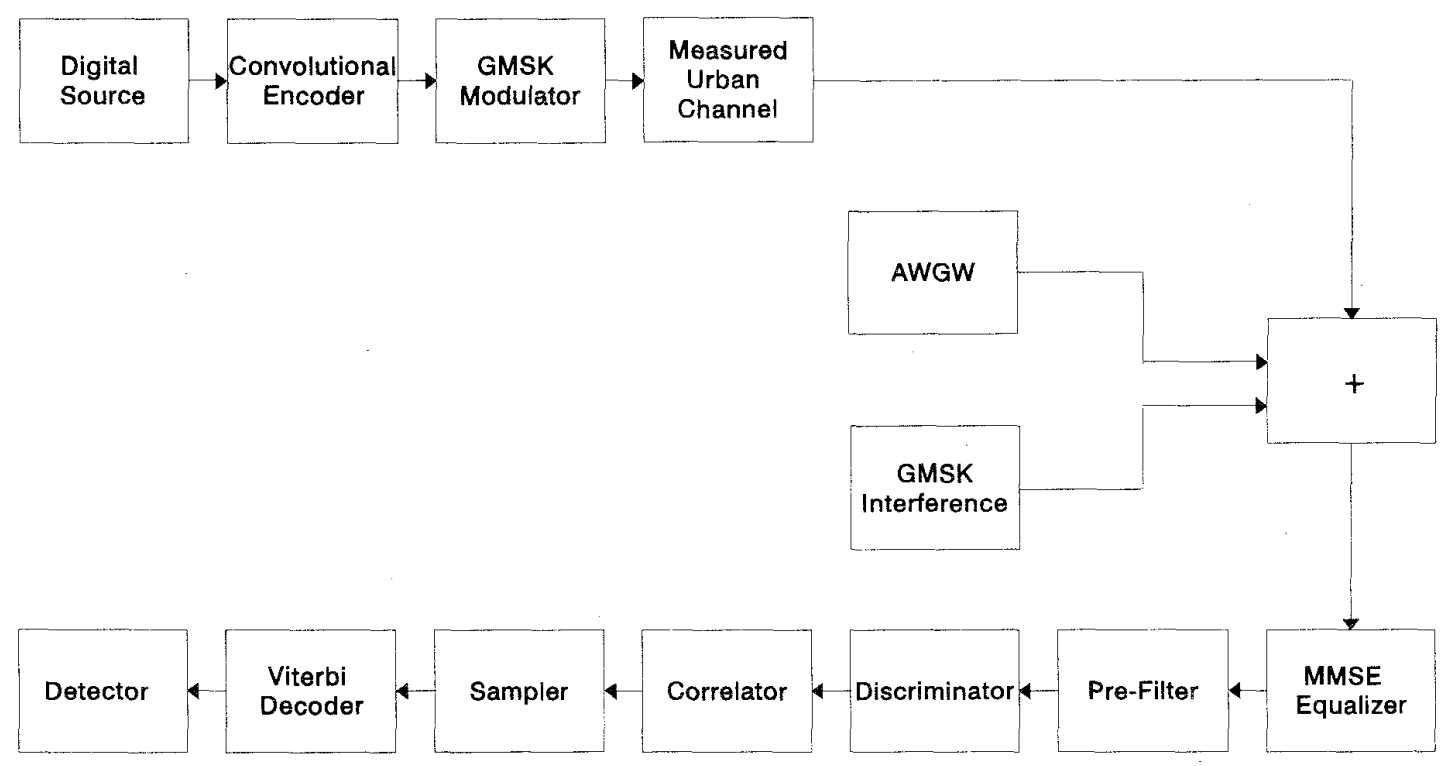

Fig. 1. GSM simulation block diagram. 


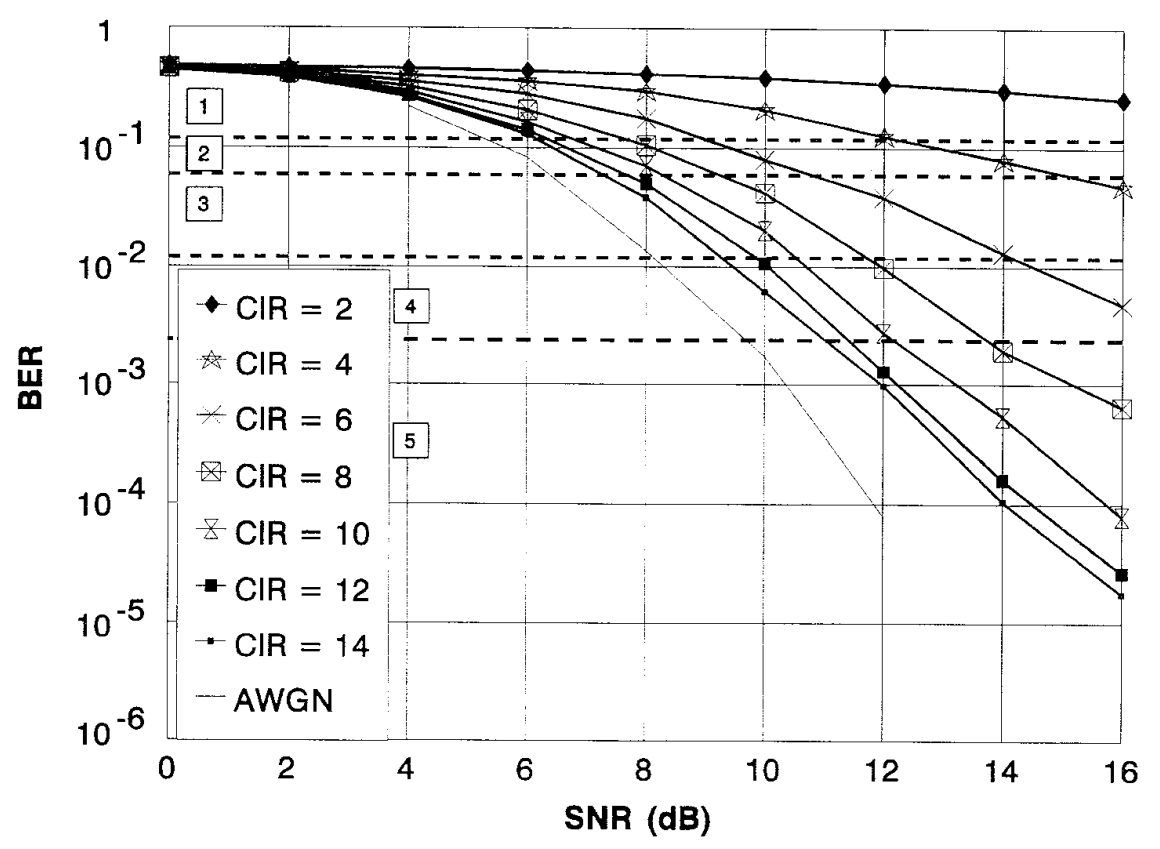

Fig. 2. GSM performance relating vocoded speech quality to system and channel parameters [GMSK modem, GMSK interference, 5 path urban channel, data rate $=270.833 \mathrm{kbps}$ ].

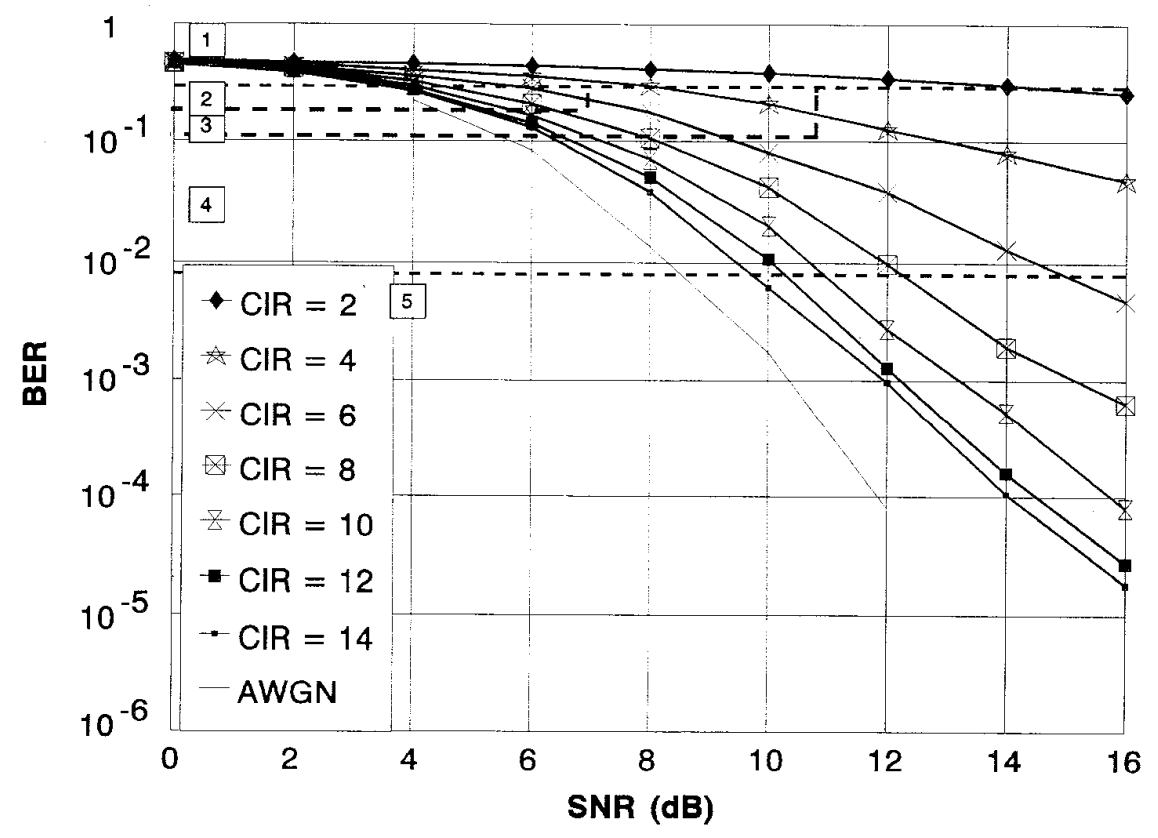

Fig. 3. GSM performance relating image quality to system and channel parameters [GMSK modem, GMSK interference, 5 path urban channel, data rate = $270.833 \mathrm{kbps}]$. 

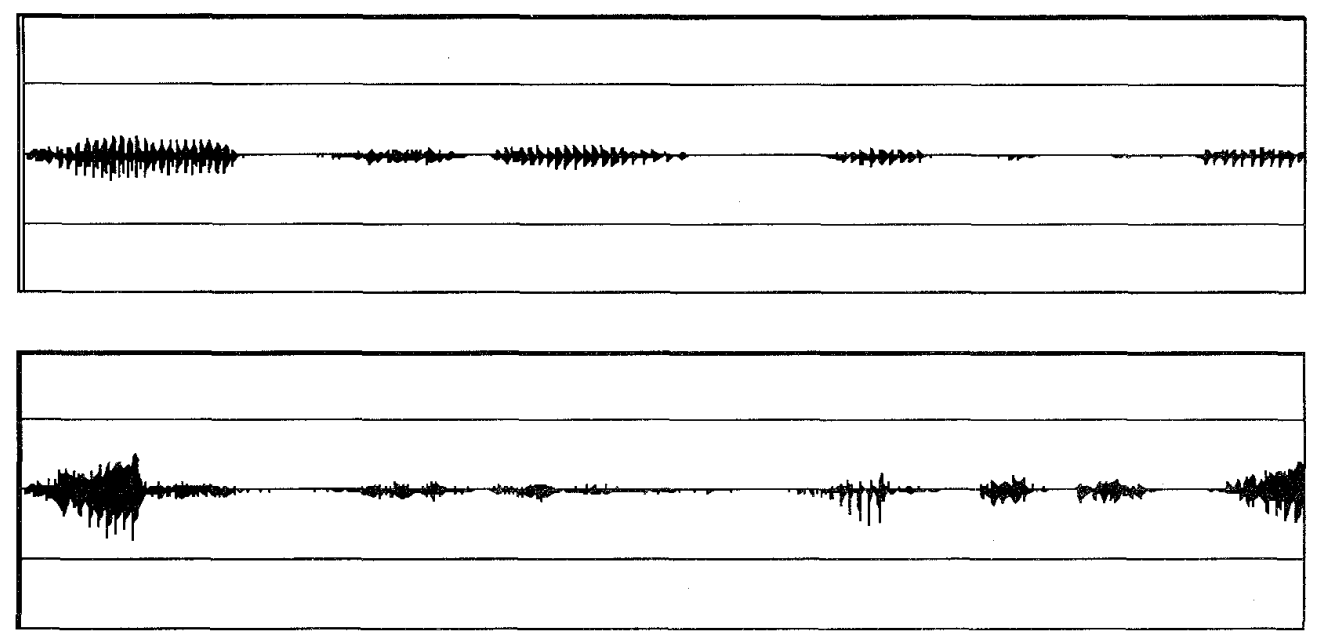

Fig. 4. GSM vocoded speech quality class examples for GMSK modem with GMSK cochannel interference: (a) Top --- Class $5(\mathrm{SNR}=16 \mathrm{~dB}, \mathrm{CIR}=12 \mathrm{~dB}, \mathrm{BER}=0.0000270)$, (b) Bottom -.. Class $2($ SNR $=8 \mathrm{~dB}, \mathrm{CIR}=8 \mathrm{~dB}, \mathrm{BER}=0.107)$.
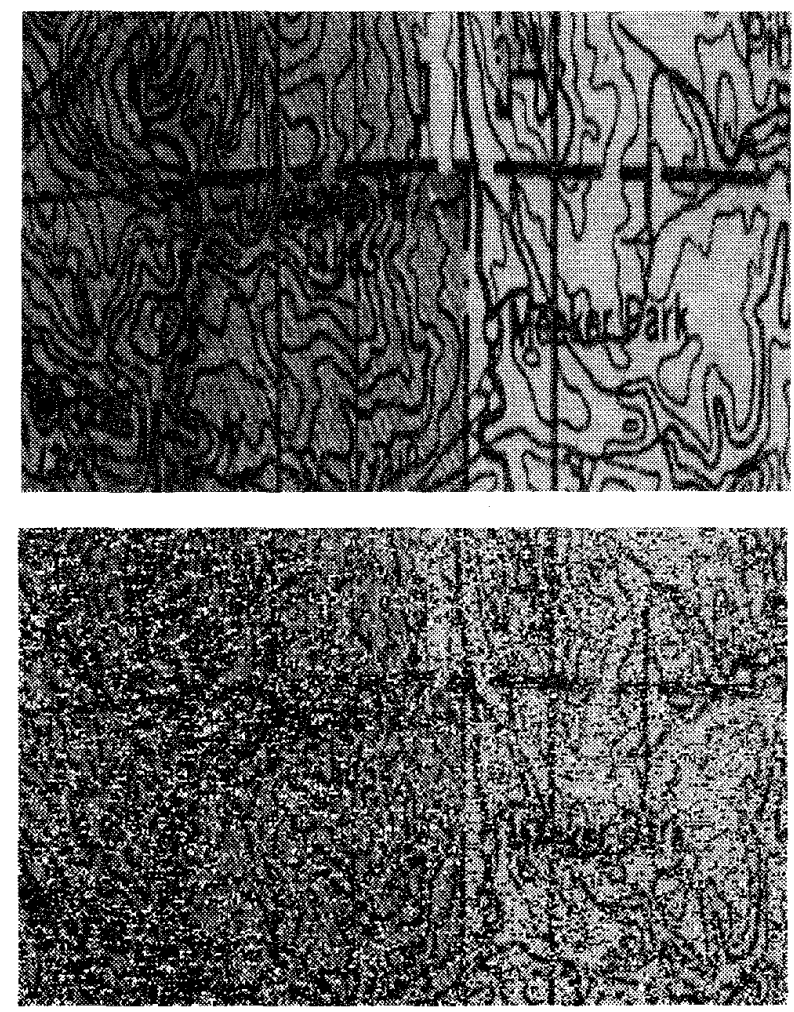

Fig 5. GSM image quality class examples for GMSK modem with GMSK cochannel interference: (a) Top --- Class $5(\mathrm{SNR}=16 \mathrm{~dB}, \mathrm{CIR}=12 \mathrm{~dB}$, BER $=0.0000270)$, (b) Bottom --- Class 2 (SNR $=6 \mathrm{~dB}, \mathrm{CIR}=8 \mathrm{~dB}, \mathrm{BER}=0.208$ ). 